\title{
A Solution Method for Low Voltage Problem in Distribution Network Considering Voltage-Violated Buses
}

\author{
Junkai $\mathrm{Ge}^{1, \mathrm{a}}$, Junqing $\mathrm{Gao}^{2, \mathrm{~b}}$, Jing Zhang ${ }^{3, \mathrm{c}}$, Tiyin $\mathrm{Li}^{1, \mathrm{~d}}$, Jiafang $\mathrm{Wu}^{4, \mathrm{e}}$ and $\mathrm{Yu}$ \\ $\mathrm{Su}^{4, \mathrm{f}}$ \\ ${ }^{1}$ Yuhang Power Supply Company of State Grid Zhejiang Hangzhou Electric Power Company, \\ Hangzhou 311100, China; \\ ${ }^{2}$ Zhejiang Truly Electric Co., Ltd., Hangzhou 311100, China; \\ ${ }^{3}$ Hangzhou Radically Electric Power Design Co., Ltd., Hangzhou 311100, China; \\ ${ }^{4}$ State Key Laboratory of Power Transmission Equipment \& System Security and New Technology, \\ Chongqing University, Chongqing 400044, China \\ a415397022@qq.com, b871965882@qq.com, '529737265@qq.com, d451592205@qq.com, \\ 6559228695@qq.com, 745328181@qq.com
}

\begin{abstract}
Keywords: voltage-violated bus, wide area reactive power control, low voltage solution, cooperative co-evolutionary
\end{abstract}

Abstract: This paper is to propose a management method of distribution network's wide-area reactive power control with low voltage-violated buses. The concept of voltage-violated bus was proposed, and based on this concept, the distribution network's reactive power control with low voltage-violated buses was established. This model was composed of the controlled voltage sub-model for weak areas and the reactive power optimization sub-model for non-weak areas. The objectives were the minimum downside voltage deviation for voltage-violated buses and the minimum real power loss respectively. The set of constraints were based on the conventional reactive power optimization constraints and including updating the lower voltage boundary constantly. In order to enhance solving-efficiency, the parallel cooperative co-evolutionary differential evolution algorithm (PCCD-EA) is hired for solving the proposed model. Finally, a distribution system with 55 buses of Hangzhou was used for simulating and verifying that the proposed method was effective.

\section{Introduction}

Some urban distribution network with large rural characteristics has a lot of $10 \mathrm{kV}$ rural feeder. The feeder or the distribution transformer reactive power compensation capacity is insufficient, and the feeder power factor is low. The magnitude and rate change of voltage and power are great [1].

Reactive power compensation for the key nodes is used to solve the low voltage problem of distribution network [2]. It achieves distribution transformer load balance of the reactive power demand in the same area. In the actual distribution network, because the $110 \mathrm{kV}$ substation voltage regulation ability is insufficient, the low voltage busbar voltage is lower. The dispersive reactive compensation configuration and insufficient capacity result in feeder voltage's drop, part of the node voltage is lower [3].

In order to control the low voltage problem caused by reactive power shortage, the main method is to take the low voltage side reactive power supply, and the wide area reactive power optimization control $[2,4]$. If the voltage of the whole distribution network can't meet, it will cause that the reactive power optimization is not feasible.

This paper takes distribution network as the research object, and studies in voltage-violated bus $[5,6]$ of the distribution network. It reduces the power loss of network, and improves security benefits of the voltage.

In this paper, the distribution network's reactive power control with low voltage-violated buses was established. A parallel cooperative evolutionary algorithm [6] is used to solve the model. 


\section{Low Voltage Problem in Distribution Network Considering Voltage-Violated Buses}

The concept of voltage-violated bus: If the voltage is not increased to a safe range by the reactive power regulation, this node is called voltage-violated bus in this paper.

The model: According to whether the node voltage is lower, the system can be divided into reactive power compensation of weak areas and reactive power compensation of non-weak areas. The control goal of the former is the minimum downside voltage deviation. The latter needs to achieve two control objectives. One is to achieve the voltage control goal of the weak areas, the other is to reduce the active power loss. From both benefit relations, the importance of the voltage control in weak areas in preference to the importance of the reactive power optimization in non-weak areas.

\section{The Reactive Power Compensation Voltage Control Model}

Weak Areas Model. The weak area voltage control method can be divided into two aspects. 1) The reactive power compensation of weak areas all input. Through the multiple load distribution transformer variable ratio adjustment, it can realize reactive power regulation of the weak areas. 2) Comprehensive adjustment of the regulating equipment in non-weak areas. The objective function of the voltage control model:

$$
S_{j}=\max \left\{V_{\min , i}-V_{\text {violated }, i}\right\} \quad i \in \text { weak area } j
$$

Type: $S_{j}$ indicates the downside voltage deviation for voltage-violated buses; $V_{\text {violated }, i}$ indicates the voltage amplitude of voltage-violated bus $i ; V_{\min , i}$ indicates the lower voltage boundary of voltage-violated bus $i$.

$$
u_{s, j}=\frac{S_{j}}{V_{\min , i}} \times 100 \%
$$

Type: $u_{s, j}$ indicates the voltage deviation percentage.

The objective function of the voltage control model for weak area $j$ is the minimum $u_{s, j}$.

If weak area $j$ is provided with load regulating transformers, the control variables of the high-pressure side are the equivalent ideal ratio, and number is the number of load regulating transformers. The specific voltage control model for type (3) (11). If there is no load regulating transformer, the model for type (3) (9) and type (11).

$$
\begin{aligned}
& \min f_{1}=u_{s, j} \\
& \text { s.t. } \quad g_{P i}(x)=P_{G i}-P_{D i}-P_{i}=0 \quad i=1, \mathrm{~K}, N_{b} \text { 且 } i \neq i_{s} \\
& g_{Q i}(x)=Q_{G i}+Q_{c r i}-Q_{D i}-Q_{i}=0 \quad i=1, \mathrm{~K}, N_{b} \\
& Q_{G i \min } \leq h_{G i}(x)=Q_{G i} \leq Q_{G i \max } \quad i=1, \mathrm{~K}, N_{G} \\
& V_{G i \min }^{2} \leq h_{V G i}(x)=e_{i}^{2}+f_{i}^{2} \leq V_{G i \max }^{2} \quad i=1, \mathrm{~K}, N_{G} \\
& h_{V i}(x)=e_{i}^{2}+f_{i}^{2} \leq V_{i \max }^{2} \quad i=1, \mathrm{~K}, N_{p q} \\
& h_{V i}(x)=e_{i}^{2}+f_{i}^{2} \geq V_{i \min }^{2} \quad i=1, \mathrm{~K}, N_{p q} \\
& k_{t \min } \leq h_{k t}(x)=k_{\text {weak }, t} \leq k_{t \max } \\
& Q_{c r i}=Q_{c r i \max } \quad i \in \text { weak area } j
\end{aligned}
$$

Non-weak Areas Model. Keep the voltage amplitude of voltage-violated bus $m$ in weak area $j$ is greater than or equal to $V_{w j, m}$, and optimize the control variables of non-weak areas. The objectives are the minimum active power loss and the minimum downside voltage deviation for voltage-violated buses. Non-weak areas reactive power optimization model:

$$
\min f_{2,1}=P_{\text {loss }}
$$




$$
\begin{aligned}
& \min f_{2, j}=u_{s, j} \quad j=1,2, \mathrm{~K}, N_{\text {weak }, s} \\
& \text { s.t. } \quad g_{P i}(x)=P_{G i}-P_{D i}-P_{i}=0 \quad i=1, \mathrm{~K}, N_{b} \text { 且 } i \neq i_{s} \\
& g_{Q i}(x)=Q_{G i}+Q_{c r i}-Q_{D i}-Q_{i}=0 \quad i=1, \mathrm{~K}, N_{b} \\
& V_{G i \min }^{2} \leq h_{V G i}(x)=e_{i}^{2}+f_{i}^{2} \leq V_{G i \max }^{2} \quad i=1, \mathrm{~K}, N_{G} \\
& h_{V i}(x)=e_{i}^{2}+f_{i}^{2} \leq V_{i \max }^{2} \quad i=1, \mathrm{~K}, N_{p q} \\
& h_{V i}(x)=e_{i}^{2}+f_{i}^{2} \geq V_{i \min }^{2} \quad i=1, \mathrm{~K}, N_{p q} \\
& k_{t \min } \leq h_{k t}(x)=k_{t} \leq k_{t \max } \quad i=1, \mathrm{~K}, N_{\text {unweak }, T} \\
& Q_{c r i \min } \leq h_{c r i}(x)=Q_{c r i} \leq Q_{c r i \max } \quad i=1, \mathrm{~K}, N_{\text {unweak }, \mathrm{cr}} \\
& h_{V i}(x)=e_{i}^{2}+f_{i}^{2} \geq V_{w j, m}^{2} \quad j=1, \mathrm{~K}, N_{\text {weak }, \mathrm{s}}, \text { me weak area } j
\end{aligned}
$$

\section{Solving Model}

The Structure of the Model. The distribution network's reactive power control with low voltage-violated buses is established by combining type (3) (11) and type (12) (21). This model is composed of the controlled voltage sub-model for weak areas and the reactive power optimization sub-model for non-weak areas.

Parallel Cooperative Evolutionary Algorithm. In this paper, we use the parallel cooperative evolutionary algorithm to solve the model. For different objective function characteristics of sub-model I and II, the fitness function of the weak areas voltage control model is defined:

$$
\text { fitness }=1 / f_{1}
$$

By using the relative dominant strategy, the fitness function of the non-weak areas reactive power optimization model is defined:

$$
\begin{aligned}
& F=f_{2,1} / f_{2,1}^{\min }+\sum_{j=1}^{N_{\text {weak.s. }}} f_{2, j} / f_{2, j}^{\min } \\
& \text { fitness }=1 / F
\end{aligned}
$$

The specific calculation steps are as follows: 1) the methods of power distribution partition and short circuit impedance distance reactive power partition are used to control the distribution network reactive power partition. 2) Use the voltage reactive hierarchical partitioning heuristic adjustment method, and determine the partition with the lower limit voltage node. These partitions are identified as the initial weak area. 3) In the cooperative evolutionary computation, the weak area population is calculated preferentially, and the control scheme with the minimum voltage deviation percent of the voltage-violated bus in the weak area is obtained. 4) If the voltage-violated buses satisfy the bounds on the updating value, it will have the optimization calculation in the non-weak area population, and get the non-weak area control scheme with the lowest active power loss in non-weak areas and the maximum voltage benefit in weak areas. 5) When parallel cooperative evolutionary algorithm iterates maximum evolution algebra, the algorithm is convergent. The calculation is end.

\section{Example Analysis}

Based on the method proposed in this paper and the R2012a Matlab software, the research on the wide-area reactive power control of a practical $10 \mathrm{kV}$ rural distribution network in Hangzhou was carried out. An actual $10 \mathrm{kV}$ distribution network consists of 55 buses, the specific connection mode is shown in Figure 1. This paper sets the reference power for 100kVA and the reference voltage is $10 \mathrm{kV}$.The distribution network is divided into 3 areas. A2 and A3 are the initial reactive power compensation weak areas. Figure 2 shows each node voltage amplitude curves of the actual 10kV 55 buses distribution network in the initial state and after optimization. Table 1 shows the partial voltage optimization results of the reactive power compensation weak area A2 and A3. 
In this paper, the model can be used to regulate the voltage of the distribution network effectively, and reduce the number of voltage-violated buses significantly, and solve the low voltage problem of the node.

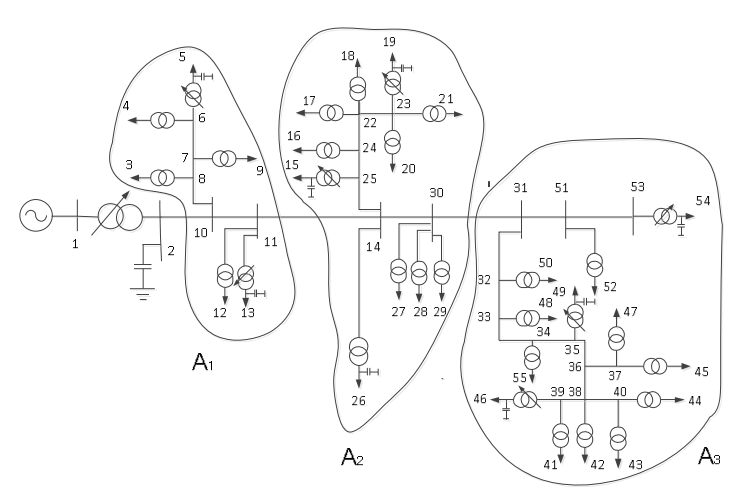

Fig. 1 A 55-bus distribution system of Hangzhou

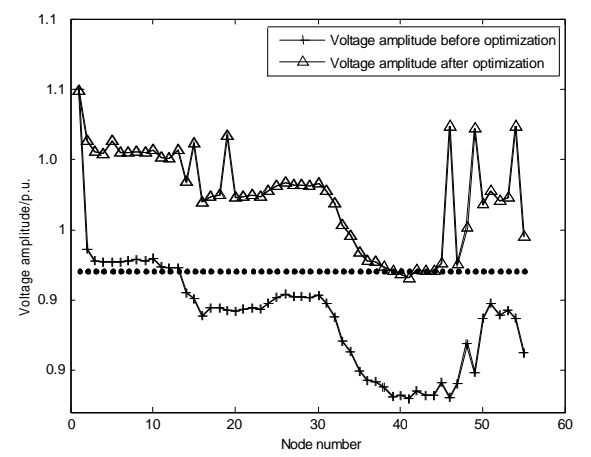

Fig. 2 Voltage magnitude for 55-bus distribution system nodes

Tab.1 The partial solution of the proposed model (p.u.)

\begin{tabular}{ccccc}
\hline $\begin{array}{c}\text { Distribution transformer } \\
\text { low voltage side node } \\
\text { number }\end{array}$ & $\begin{array}{c}\text { Location } \\
\text { partition }\end{array}$ & $\begin{array}{c}\text { Voltage amplitude } \\
\text { before optimization }\end{array}$ & $\begin{array}{c}\text { Voltage amplitude } \\
\text { after optimization }\end{array}$ & $\begin{array}{c}P_{\text {loss }} \text { before } \\
\text { optimization }\end{array}$ \\
\hline 16 & $A_{2}$ & 0.9386 & 1.0192 & \\
optimization
\end{tabular}
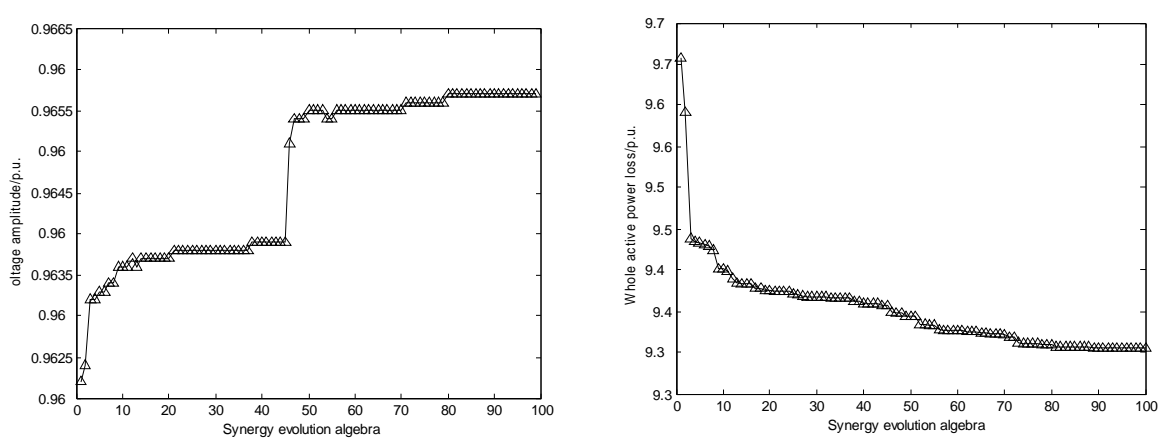

(a) Voltage rise curve of voltage-violated buses in A3 (b) Active network loss decline curve

Fig. 3 The convergence curve of IPCCD-EA

Figure 3 (a) and (b) show that IPCCD-EA algorithm searches voltage-violated buses voltage amplitude rise process along with the network loss decreased. 


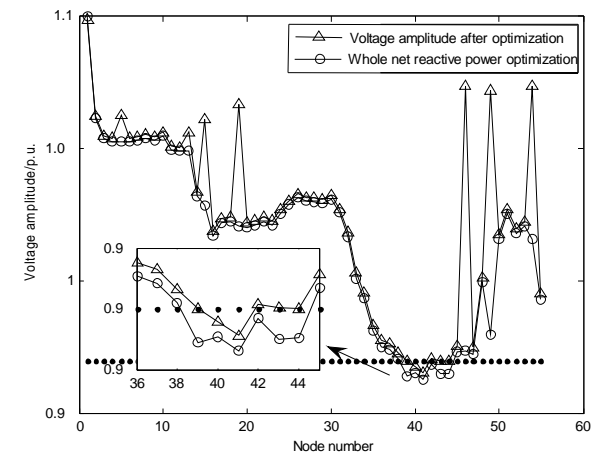

Fig. 4 Comparison of the results of the method and reactive power optimization

Use the reactive power optimization calculation method with the voltage amplitude constraints to $[0.9,1.07]$, and get active power loss is 9.2031 p.u., while the active power loss is 9.3552 p.u.by using the method in this paper. The number of voltage-violated buses is 6 , and it could reduce to 2 by using the method in this paper. The specific results shown in Figure 4.

\section{Conclusion}

In this paper, the concept of voltage-violated bus was proposed firstly, and based on this concept, the distribution network's reactive power control with low voltage-violated buses was established. This model was composed of the controlled voltage sub-model for weak areas and the reactive power optimization sub-model for non-weak areas. The method proposed in this paper solves the problem of reactive power control strategy in distribution network with non-feasible voltage.

\section{References}

[1] LU Ji-ping,YE Quan-qiang,TANG Chao,et al.Disscussion in the Rural Power Network Construction in the New Rural Construction[J].Journal of Chongqing University,2007,27 ( 24 ) :63-66(in Chinese).

[2] YAN Wei,ZHAO Ke,ZHAO Xia,et al.Wide-Area Reactive Optimal Control Method of Radial Distribution Networks Based on Partial Measurement Data [J].Power System Protection and Control, 2014, 42(1): 47-53.

[3] ZHOU Hu,CHEN JiaLi,XIAN Long,et al.Research on the low voltage of three-phase unbalance in rural Grid [J].Power System and Clean Energy, 2015, 11(04): 47-53.

[4] ZHOU Yun-cheng,PIAO Zai-lin,FU Li-si,et al.Design of reactive power optimization automatic control system for $10 \mathrm{kV}$ distribution systems[J].Power System Protection and Control,2011,39(2):125-130(in Chinese).

[5] Ding Qia,Li Naihu,Wu Han.Infeasibility study on voltage/reactive power optimization[J].Power System Technology,1999,23(9):19-22(in Chinese).

[6] LEI Chao.A recognizing method for weak buses for the infeasibility of reactive optimization problem [D].Chongqing:Chongqing University,2015(in Chinese). 